\title{
A Case of Thrombosis and Thromboembolic Events
}

Daniel Lin

Thomas Jefferson University

Follow this and additional works at: https://jdc.jefferson.edu/tmf

Part of the Cardiology Commons

Let us know how access to this document benefits you

\section{Recommended Citation}

Lin, Daniel (2003) "A Case of Thrombosis and Thromboembolic Events," The Medicine Forum: Vol. 4 , Article 8.

DOI: https://doi.org/10.29046/TMF.004.1.009

Available at: https://jdc.jefferson.edu/tmf/vol4/iss $1 / 8$

This Article is brought to you for free and open access by the Jefferson Digital Commons. The Jefferson Digital Commons is a service of Thomas Jefferson University's Center for Teaching and Learning (CTL). The Commons is a showcase for Jefferson books and journals, peer-reviewed scholarly publications, unique historical collections from the University archives, and teaching tools. The Jefferson Digital Commons allows researchers and interested readers anywhere in the world to learn about and keep up to date with Jefferson scholarship. This article has been accepted for inclusion in The Medicine Forum by an authorized administrator of the Jefferson Digital Commons. For more information, please contact: JeffersonDigitalCommons@jefferson.edu. 


\section{A Case of Thrombosis and Thromboembolic Events}

Daniel Lin, Internal Medicine Resident, PGY3, 2000-2003

A 65 year old black woman with hypertension (HTN), gastroesophageal reflux disease (GERD), and anxiety had complained to her primary care physician of vague abdominal pain. Initial empiric treatment as an outpatient for worsening GERD was unsuccessful. The patient had insidious development of generalized fatigue, early satiety, nausea, vomiting, and weight loss. An ultrasound of the abdomen revealed multiple liver lesions, with the largest lesion being $2.5 \mathrm{~cm} \times 2.5 \mathrm{~cm}$. She subsequently underwent a CT scan of the abdomen and pelvis, which confirmed multiple hyperdense liver lesions. The remainder of the scan was unremarkable. Esophagogastroduodenoscopy (EGD) as well as colonoscopy was performed, revealing a duodenal mass and a sigmoidal polyp. Biopsy of these two lesions showed an adenomatous polyp and a tubular adenoma, respectively, and subsequent liver biopsy diagnosed poorly differentiated adenocarcinoma. The patient was then admitted to TJUH for further management.

Past medical and surgical history included HTN, GERD, anxiety, and total hysterectomy. She had no known drug allergies. The patient denied current or prior use of tobacco, alcohol, and illicit drugs. She was a retired first grade teacher. Her family history was significant only for coronary artery disease and hypertension.

Her outpatient medications included metoprolol, fosinopril, valsartan, atorvastatin, lansoprazole, lorazepam, and Percocet.

Review of systems was significant for lack of urinary symptoms, changes in bowel habits, recent skin changes, dysphagia, cough, or shortness of breath. She also denied fevers, chills, and sweats. However, her sister felt that the patient recently began having intermittent episodes of confusion and new speech difficulties.

The patient was afebrile with a temperature of $97.7^{\circ} \mathrm{F}$, blood pressure of $146 / 73 \mathrm{~mm} \mathrm{Hg}$, pulse of $90 / \mathrm{min}$, and respiratory rate of $22 / \mathrm{min}$. Head and neck exam was unremarkable. Cardiac examination revealed a regular rate and rhythm with no murmurs, rub, or gallops. Her lungs were clear to auscultation. Palpation of the abdomen revealed a $3 \mathrm{~cm} \times 3 \mathrm{~cm}$ mobile tender mass in the right lower quadrant. Bowel sounds were present and normal. Rectal examination revealed normal tone and brown stool that was hemoccult positive. Her extremities were non-edematous, and her skin without rashes or changes in pigmentation. Neurologic examination was significant for a right homonymous hemianopsia. The remainder of her cranial nerves were intact. Reflexes were equal and symmetric. No pronator drift or Babinski sign was identified. Her strength was $5 / 5$ in all extremities. A mini-mental status exam revealed difficulty with repetition and naming, as well as perseveration.

The results of her blood work are listed in Table 1. A repeat computerized tomographic (CT) scan of the thorax, abdomen, and pelvis was performed, which revealed bibasilar atelectasis without effusion or lymphadenopathy. CT of the abdomen and pelvis showed numerous variable- sized lesions in both lobes of the liver, bilateral wedge shaped infarcts in the kidneys, and a right common femoral vein thrombus. The remainder of the abdomen and pelvis was unremarkable. Magnetic resonance imaging (MRI) of the head revealed multiple areas of infarction (Figure 1). Both cerebral hemispheres were involved, primarily involving the distribution of the left posterior cerebral artery (PCA) and left middle cerebral artery (MCA). Magnetic resonance angiography (MRA) of the head was negative for vascular abnormalities.

The patient underwent transthoracic echocardiography, which showed dynamic left ventricular function, dilated right ventricle with hypokinesis, mild mitral regurgitation, moderate tricuspid regurgitation, and severe pulmonary hypertension with a RV-RA gradient of $50 \mathrm{mmHg}$. Also noted on echocardiography were vegetations on the anterior and posterior leaflets of the mitral valve. Further serologic studies were performed and the results are shown in Table 2. Three sets of blood cultures failed to grow an organism.

\section{Differential Diagnosis}

The differential diagnosis for this patient was made for thrombosis and thromboembolic phenomena. Ideally, a 
unifying diagnosis should explain both the deep venous thrombosis (DVT) as well as the multiple cerebral and renal infarctions suffered by the patient. The differential diagnosis list for thrombotic disorders is lengthy and is beyond the scope of this article, but includes coagulation factor disorders or deficiency, impaired clot lysis, malignancy, myeloproliferative disorders, hyperviscosity states, antiphospholipid syndrome, thrombotic thrombocytopenic purpura, disseminated intravascular coagulation, and many others. This extensive list can be shortened, given several facts about this case. First, the cerebral and renal infarctions are most likely due to embolic phenomena, rather than in-situ thrombosis. Second, vegetations were identified on echocardiogram. Third, there was no supporting evidence by personal or family history that the patient would be harboring a genetic defect predisposing her to thrombosis. The patient's major risk factor for DVT and venous thrombosis was her adenocarcinoma. Historically, mucin-producing neoplasms have offered the greatest risk for thromboembolic complications. This is the most likely reason for her DVT. But how do we explain the multiple infarctions in the arterial system? The distribution of the areas of infarct is typical of an embolic mechanism, with the heart the most likely source. However, one must consider the possibility of paradoxical embolism, atrial myxoma, and tumor emboli. Paradoxical embolism is unlikely in this case, as no pulmonary emboli were suspected or identified. While a patent foramen ovale may allow venous thrombotic fragments to enter the arterial circulation, it would not preclude pulmonary emboli to occur. Thus one would expect to find at least several pulmonary emboli, given the high number of arterial emboli seen. Tumor emboli are most commonly seen with lung cancers entering the pulmonary venous system and embolizing systemically. In our patient, no atrial myxoma or primary tumor could be found, though a gastrointestinal primary was most likely. Since the echocardiogram revealed vegetations on the mitral valve, these are the most likely source of the patient's systemic infarctions. No organism was recovered from blood cultures, and the patient remained clinically stable, without fevers or hemodynamic compromise, even without administration of antibiotics. The constellation of metastatic adenocarcinoma, DVT, valvular vegetations, embolic phenomena, and negative blood cultures lead to the diagnosis of marantic endocarditis.

\section{Discussion}

Marantic endocarditis is known by many names, including nonbacterial thrombotic endocarditis (NBTE), degenerative verrucal endocarditis, terminal endocarditis, endocardiosis, and endocarditis simplex. Classically, NBTE has been associated with cancer. It has been reported in association with almost every cancer, with the exception of brain cancer. Over $50 \%$ of NBTE cases are associated with malignant neoplasms. The most commonly associated cancers with NBTE are pancreas and lung. Many nonneoplastic diseases are also associated with NBTE, albeit a much smaller percentage of cases. These include rheumatic heart disease, systemic lupus erythematosus, ulcerative fibrosis, membranoproliferative glomerulonephritis, polyarteritis nodosa, and many others. NBTE has also been associated with disseminated intravascular coagulation (DIC), and their pathologic mechanisms have been thought to be similar.

There is no gender bias for development of NBTE. It is seen more commonly in patients during the fourth through eighth decades of life. However, it has been seen in all ages, including infants and children. The finding of NBTE, though rare in the living, has been estimated at $0.3 \%$ up to $9.3 \%$ of autopsies.

The pathogenesis of NBTE has been theorized to require valvular irregularity, with subsequent thrombus formation on the irregular valve surface. The valve often appears grossly normal. This results in a nonbacterial or sterile vegetation that also lacks inflammatory changes. Such a mechanism helps to explain the development of NBTE in patients with pulmonary artery catheters. The vegetations seen with catheter placement generally occur after 4 days duration of catheterization, and most likely involve the right sided valves. This is in contrast to noncatheter associated NBTE, which mostly develops on left sided valves. The aortic valve is affected slightly more often than the mitral valve (39.3\% vs. $37.3 \%)$. 
It is often difficult to identify NBTE due to lack of symptoms until embolism occurs. Embolic phenomena occur in approximately $42 \%$ of cases, with a reported range of $14 \%$ to $91 \%$. The higher rates of embolism are seen in neoplasm-associated NBTE. Cerebral infarction is the most common (80\%), followed by renal and splenic injury. However, myocardial, mesenteric, extremity, and skin infarctions may also be seen. Patients with embolic complications secondary to NBTE are often asymptomatic, though they may develop chest pain, abdominal pain, mental status changes, and hematuria.

Valvular dysfunction can also occur with NBTE, but it is not known whether valvular dysfunction leads to vegetation formation, or if vegetations cause valvular dysfunction. Most often, surgery is indicated to repair and/or replace the dysfunctional valve, at which time NBTE is frequently diagnosed.

\section{Diagnosis}

Diagnosis of NBTE is based on historical and clinical findings. M-mode echocardiography has not been proven to be useful in diagnosing NBTE, because many vegetations may be less than $2 \mathrm{~mm}$ in size and easily missed. Also vegetations are composed only of platelets and fibrin, and may easily and completely dislodge with embolization. It is important to eliminate infectious endocarditis (IE) as an etiology. Blood cultures should be drawn, and special media and prolonged incubation used, if culture-negative endocarditis is suspected. Head imaging with angiography is important, since mycotic aneurysms complicate $4 \%$ to $10 \%$ of IE cases. Mycotic aneurysms may be found in any vessel, though tend to involve the distal middle cerebral artery, and are multiple and bilateral. The overall mortality of patients with a mycotic aneurysm is $60 \%$ and rises to $80 \%$ with rupture. Risk of rupture is increased with anticoagulation therapy, as is risk for intracerebral hemorrhage (ICH) with cardioembolic strokes.

\section{Treatment}

There is no proven effective treatment for NBTE. Once NBTE and DIC were felt to represent different entities on a clinical spectrum, and that treatments beneficial for DIC would also treat NBTE. No trials of anticoagu- lation with heparin or warfarin have shown benefit in treating NBTE, despite theories that anticoagulation would prevent platelet and fibrin deposition on valves.6 Management of NBTE involves symptomatic and supportive therapy.

In our patient, the risk of anticoagulation for DVT had to be weighed against the risk of ICH after embolic strokes. It was felt that the benefit of anticoagulation for DVT far outweighed the risk of $\mathrm{ICH}$, and the patient was begun on enoxaparin injections.

Table 1. Initial Laboratory Values

\begin{tabular}{|c|c|c|c|}
\hline Laboratory & Result & Normal & Units \\
\hline WBC & 19.2 & $4-11$ & 103 cells $/ \mathrm{cm} 3$ \\
\hline Neutrophils & 81 & $40-73$ & $\%$ \\
\hline Bands & 8 & $0-9$ & $\%$ \\
\hline Lymphocytes & 5 & $20-44$ & $\%$ \\
\hline $\mathrm{Hgb}$ & 9.1 & $12.5-15$ & $\mathrm{~g} / \mathrm{dL}$ \\
\hline Platelets & 227 & $140-400$ & 103 cells $/ \mathrm{cm} 3$ \\
\hline Reticulocytes & 4 & $0.5-1.5$ & $\%$ \\
\hline $\mathrm{Na}$ & 142 & $135-146$ & $\mathrm{mmol} / \mathrm{L}$ \\
\hline K & 3.6 & $3.5-5.0$ & $\mathrm{mmol} / \mathrm{L}$ \\
\hline$\overline{\mathrm{Cl}}$ & 103 & $98-109$ & $\mathrm{mmol} / \mathrm{L}$ \\
\hline $\mathrm{HCO} 3$ & 24 & $24-32$ & $\mathrm{mmol} / \mathrm{L}$ \\
\hline BUN & 17 & $7-26$ & $\mathrm{mg} / \mathrm{dL}$ \\
\hline Creatinine & 0.9 & $0.7-1.4$ & $\mathrm{mg} / \mathrm{dL}$ \\
\hline Glucose & 145 & 60-110 & $\mathrm{mg} / \mathrm{dL}$ \\
\hline $\mathrm{iCa}$ & 4.5 & $4.5-5.3$ & $\mathrm{mg} / \mathrm{dL}$ \\
\hline $\mathrm{Mg}$ & 2.1 & $1.3-2.1$ & $\mathrm{mg} / \mathrm{dL}$ \\
\hline PO4 & 3.5 & $2.5-4.5$ & $\mathrm{mg} / \mathrm{dL}$ \\
\hline Protein & 6.8 & $6.0-8.5$ & $\mathrm{~g} / \mathrm{dL}$ \\
\hline Albumin & 3.3 & $3.2-4.9$ & $\mathrm{~g} / \mathrm{dL}$ \\
\hline$\overline{\mathrm{AST}}$ & 68 & $7-35$ & IU/L \\
\hline ALT & 48 & $1-30$ & IU/L \\
\hline Alk. Phos. & 607 & $25-120$ & IU/L \\
\hline Bilirubin, Total & 3.1 & $0.2-1.2$ & $\mathrm{mg} / \mathrm{dL}$ \\
\hline Bilirubin, Direct & 1.3 & $0.0-0.4$ & $\mathrm{mg} / \mathrm{dL}$ \\
\hline $\mathrm{LDH}$ & 347 & $100-200$ & IU/L \\
\hline Ammonia & 46 & $11-35$ & $\mathrm{mcmol} / \mathrm{L}$ \\
\hline Haptoglobin & 380 & $16-200$ & $\mathrm{mg} / \mathrm{mL}$ \\
\hline Iron & 26 & $40-155$ & $\mathrm{mcg} / \mathrm{dL}$ \\
\hline TIBC & 250 & $250-400$ & $\mathrm{mcg} / \mathrm{dL}$ \\
\hline Ferritin & 925 & $10-160$ & $\mathrm{ng} / \mathrm{mL}$ \\
\hline INR & 1.55 & 0.91-1.09 & N/A \\
\hline $\begin{array}{l}\text { PTT } \\
\end{array}$ & 35 & $25-37$ & $\mathrm{sec}$ \\
\hline TSH & 0.34 & $0.4-4.8$ & $\mathrm{mIU} / \mathrm{mL}$ \\
\hline FT4 & 1.3 & $0.7-1.6$ & $\mathrm{ng} / \mathrm{dL}$ \\
\hline FT3 & 227 & $230-420$ & $\mathrm{pg} / \mathrm{L}$ \\
\hline B12 & 2826 & $200-900$ & $\mathrm{pg} / \mathrm{mL}$ \\
\hline Folate & 9.1 & $3-18$ & $\mathrm{ng} / \mathrm{mL}$ \\
\hline
\end{tabular}


Table 2

\begin{tabular}{l|l|l|l}
\hline Laboratory & Result & Normal & Units \\
\hline DRVVT & 41.2 & $21.1-36.7$ & $\mathrm{sec}$ \\
\hline Mix DRVVT & 1.05 & $<1.12$ & N/A \\
\hline $\mathrm{HBsAg}$ & $\mathrm{Neg}$ & $\mathrm{Neg}$ & $\mathrm{N} / \mathrm{A}$ \\
\hline $\mathrm{HBsAb}$ & $\mathrm{Neg}$ & $\mathrm{N} / \mathrm{A}$ & $\mathrm{N} / \mathrm{A}$ \\
\hline $\mathrm{HBcAb}$ & $\mathrm{Pos}$ & $\mathrm{Neg}$ & $\mathrm{N} / \mathrm{A}$ \\
\hline $\mathrm{HCAb}$ & $\mathrm{Neg}$ & $\mathrm{Neg}$ & $\mathrm{N} / \mathrm{A}$ \\
\hline RPR & $\mathrm{Neg}$ & $\mathrm{Neg}$ & $\mathrm{N} / \mathrm{A}$ \\
\hline $\mathrm{CEA}$ & 16.9 & $0-5$ & $\mathrm{ng} / \mathrm{mL}$ \\
\hline CA125 & 2366.7 & $0-35$ & $\mathrm{U} / \mathrm{mL}$ \\
\hline CA19-9 & 5.0 & $0.0-6.4$ & $\mathrm{IU} / \mathrm{mL}$ \\
\hline
\end{tabular}

Figure 1. MRI Head.

Note the multiple infarcts located in both cerebral hemispheres.

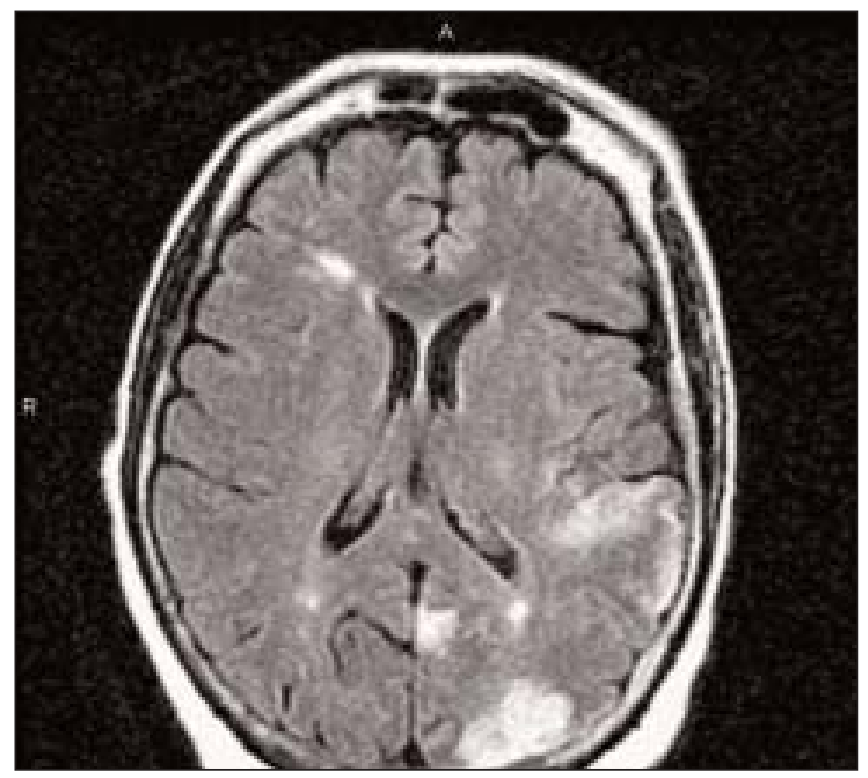

\section{References}

1. Rickles, F., et al. Activation of Blood Coagulation in Cancer: Trousseau's Syndrome Revisited. Blood 1983; 62(1): 14-31.

2. Waller, BF., et al. Marantic Valvular Vegetations. Circulation 1973; 48: 644-650.

3. Rogers, L., et al. Cerebral Infarction from Non-Bacterial Thrombotic Endocarditis. Am J Med 1987; 83: 743-756.

4. Kooiker, JC., et al. Cerebral Embolism, Marantic Endocarditis, and Cancer. Arch Neurol 1976; 33: 260-264.

5. Biller, J., et al. Nonbacterial Thrombotic Endocarditis. Arch Neurol 1982; 39: 95-98.

6. Lopez, JA., et al. Nonbacterial Thrombotic Endocarditis: A Review. Am Heart J 1987; 113(3): 773-784.

7. Lange, HW., et al. Local Complications Associated with Indwelling Swan-Ganz Catheters: Autopsy Study of 36 Cases.

8. Kuramoto, K., et al. Nonbacterial Thrombotic Endocarditis as a Cause of Cerebral and Myocardial Infarction. Jpn Circ J 1984; 48: 1000-1006.

9. Edoute, Y., et al. Cardiac Valvular Vegetations in Cancer Patients: A Prospective Echocardiographic Study of 200 Patients. Am J Med 1997; 102: 252-258.

10. Eiken, PW., et al. Surgical Pathology of Nonbacterial Thrombotic Endocarditis in 30 Patients, 1985-2000. Mayo Clin Proc 2001; 76: 1204-1212.

11. Barami, K., Ko, K. Ruptured Mycotic Aneurysm Presenting as an Intraparenchymal Hemorrhage and Nonadjacent Acute Subdural Hematoma: Case Report and Review of the Literature. Surg Neurol 1994; 41: 290-293.

12. Masuda, J., et al. Histopathological Analysis of the Mechanisms of Intracranial Hemorrhage Complicating Infective Endocarditis. Stroke1992; 23(6): 843-850.

13. Wilson, WR., et al. Management of Complications of Infective Endocarditis. Mayo Clin Proc 1982; 57: 162-170. 